\title{
De la forêt paysanne à la forêt capitaliste en pays de Sault sous l'Ancien Régime
}

L'étude de l'évolution de la hêtraie-sapinière en Pays de Sault au cours des deux derniers siècles de l'Ancien Régime a pu être réalisée grâce, essentiellement, à l'abondante documentation rassemblée à l'occasion de deux réformations pratiquées à près de quatrevingts ans d'intervalle, c'est-à-dire le temps de maturité d'un sapin. La première de ces deux réformations a concerné l'ensemble des forêts royales de France; ordonnée par Colbert, dirigée par Louis de Froidour dans le cadre de la grande maîtrise de Toulouse, elle dure en Pays de Sault de 1666 à 1673 ; la seconde, particulière à la maîtrise de Quillan, dont fait partie le Pays de Sault, dure de 1736 à 1760 , mais les travaux sur le terrain ont surtout été effectués de 1739 à 1741 . Un certain nombre d'autres documents complètent notre information (').

La situation de ces forêts peut être, ainsi, relativement bien connue, tant au XVIJe siècle qu'au XVIIJe siècle. Les procès-verbaux de visite et les plans qui les accompagnent nous renseignent sur les superficies, les essences, l'état des forêts, des sols, des arbres, la présence, la nature et l'importance des défrichements. Nous pouvons remarquer les transformations survenues d'une réformation à l'autre. D'autres documents permettent de connaître les différents milieux sociaux liés à la forêt et leur évolution.
Le Pays de Sault est un "pays " pyrénéen de $30 \mathrm{~km}$ sur 25 dans ses plus grandes dimensions, situé au sud de l'Aude, à la limite de l'Ariège. Il est constitué par un ensemble de plateaux calcaires étagés de 800 à 1200 mètres, limités à l'ouest et au sud par des hauteurs qui atteignent 1700 à 2000 mètres. La forêt est implantée sur les marges septentrionales de la région ainsi que sur les versants des montagnes de l'ouest et du sud. Dominée par les sapins, cette forêt se trouve être, aujourd'hui, une des plus belles et des plus rentables des Pyrénées.

Notre étude a eu pour but de rechercher les conditions particulières qui ont permis, dès la fin de l'Ancien Régime, la constitution d'une telle sapinière, en essayant de dépasser le cloisonnement scientifique des approches précédentes du milieu forestier - les études économiques à base juridique et administrative de M. Devèze, les perspectives naturalistes de H. Gaussen - -.. Nous avons cherché à analyser le paysage en tenant compte, à la fois, du dynamisme naturel des différentes espèces végétales et des interventions humaines, ellesmêmes replacées dans leurs contextes économique et social.

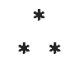

La documentation rassemblée par les officiers réformateurs à la recherche de bois pour la

(1) Autre communication "Les forêts du Pays de Sault dans les dépôts d'archives ". 


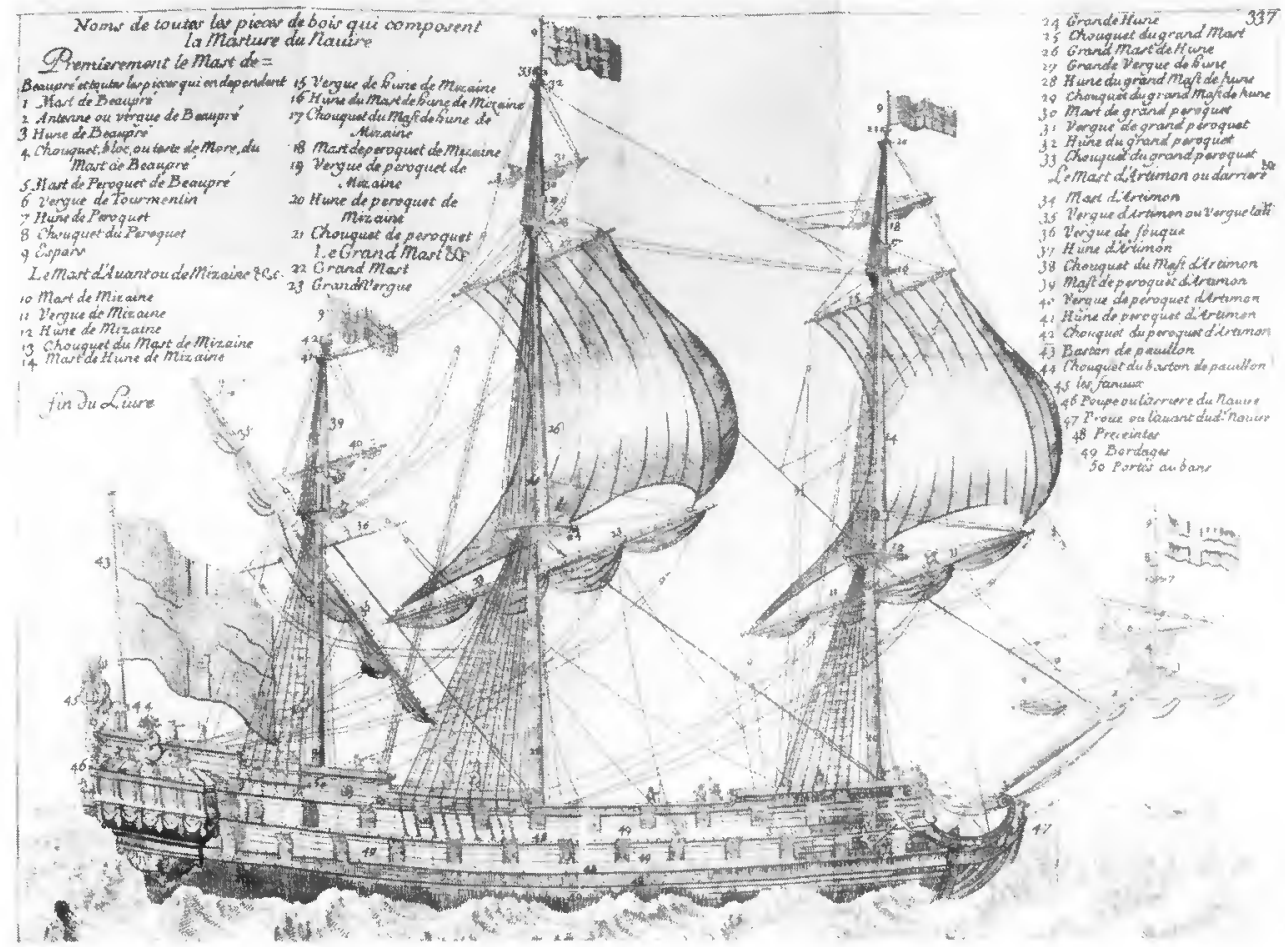

Traité des bois servant à tous usages de Claude CARON. Paris MDCLXXVI. Photo Ch. ANDRÉ.

marine et les finances royales privilégie les éléments rentables dans les forêts. Les officiers sont à la recherche de "belles » forêts et leurs jugements esthétiques sont, en fait, des jugements de nature économique. Une "belle» forêt est une forêt dont les arbres peuvent fournir des bois de marine ou de construction. Les renseignements fournis concernent donc, essentiellement, le sapin. Selon les cas, le hêtre est mentionné ou négligé, mais les données qui le concernent sont moins précises. Quant aux autres essences, elles sont désignées par des termes vagues, "pins sauvages ", " chênes " ou collectifs, " bois blancs" (noisetier, tilleul, orme, frêne, tremble) et elles n'apparaissent qu'exceptionnellement, tout comme le buis, la seule espèce du sous-bois dont il soit question.

Les renseignements fournis par les officiers réformateurs de 1666 restent encore superficiels. Les officiers décrivent l'espace forestier en opposant des ensembles : il y a la partie haute et la partie basse, plus de hêtres que de sapins ou, plus rarement, l'inverse. Les nombres sont inexistants ou difficiles à utiliser : les superficies sont calculées avec une grande précision, mais selon une méthode incorrecte en montagne. Les densités ne peuvent être calculées que dans de rares cas et en se livrant à des calculs aléatoires. Les nombres du $\mathrm{XVIII}^{\mathrm{e}}$ siècle sont plus fiables. Dans toutes les forêts, les sapins de plus de $30 \mathrm{~cm}$ de diamètre ont fait l'objet d'un comptage, triage par triage.

Dans la forêt du XVII' siècle, le hêtre domine le sapin et chacune de ces deux essences occupe une aire propre : le hêtre est installé dans la partie basse de la forêt, où il se présente à l'état de taillis très dégradé ; le sapin se mêle au hêtre vers le milieu de la forêt et domine nettement dans la partie supérieure, quelle que soit, au demeurant, l'altitude supérieure atteinte par la forêt. La futaie de hêtres ou, plus fréquemment, de sapins se trouve dans les secteurs les plus reculés et les arbres y sont clairsemés. Pour la forêt de Niave, nos calculs nous fournissent un maximum de 150 sapins à l'hectare. Certes, il s'agit là, uniquement, des sapins susceptibles de fournir des bois de construction, puisque les sapins « impropres", fourchus, branchus, jumeaux, beaucoup plus nombreux à l'époque qu'aujourd'hui, n'étaient pas comptés. Mais de tels arbres s'épanouis- 
sent, précisément, dans les forêts claires. Les jeunes pousses sont souvent nombreuses; on trouve aussi de vieux sapins, de cent cinquante jusqu'à trois cents ans ; par contre, les arbres de quatre-vingts à cent ans sont les moins nombreux, car ils sont les plus exploités.

L'espace forestier diminue considérablement devant l'importance des défrichements qui attaquent les taillis de hêtres ou les futaies situées dans les secteurs relativement plats du nord du Pays de Sault (Picaussel, Coumefrède, vallon de la Fraiche). Ailleurs, sur des soulanes ou des espaces accidentés, les champs se mêlent aux broussailles et aux buissons de hêtres, de chênes et de "bois blancs"

Dans la société rurale traditionnelle, la forêt a moins de valeur que la terre, source de profit pour les uns, de survie pour les autres. Le bois, si abondant par rapport aux besoins, n'a pas de valeur marchande ; chacun se sert librement en forêt pour produire les nombreux objets d'une technologie du bois. Le sapin en subit les conséquences de façon tout particulièrement néfaste. Il disparaît progressivement des lieux les plus proches des villages où il est exploité sans souci de l'avenir.

Comme le sapin se reproduit par semence, le stock de graines diminue peu à peu, sans disparaître pour autant puisque des graines peuvent provenir des sapins plantés dans les parties hautes des forêts. Le bétail qui pâture, par nécessité, dans la partie basse des forêts, au printemps et en automne, mange les jeunes plants de sapin dont il est très friand. Le hêtre, par contre, qui se reproduit aussi bien par voie végétative que par semence, peut se développer dans cet espace abandonné par le sapin. Les hommes coupent aussi fréquemment le bois de hêtre pour leurs besoins en chauffage et ils transforment cette forêt en taillis où le bétail, de petite stature à l'époque, se déplace d'autant plus facilement que les souches sont coupées à un mètre de haut. Enfin, dans ces taillis clairs et ensoleillés, les sapins, essence d'ombre, éprouvent toujours plus de difficultés à se développer. Le sapin est donc relégué dans la partie supérieure de la forêt où, là encore, il subit la concurrence du bétail qui y pâture d'autant plus que le tapis herbacé est plus fourni. Enfin, la progression du commerce du sapin dans le courant du XVII e siècle a précipité la disparition de cet arbre des lieux d'accès facile.
Cette progression de la hêtraie, qui plus est sous forme de taillis, dans le cadre d'une économie rurale traditionnelle, va à l'encontre des nouveaux besoins de l'économie de marché.

Le Pays de Sault bénéficie, grâce à l'Aude navigable, de communications relativement aisées avec le Bas-Languedoc et la Méditerranée, deux espaces pauvres en bois et gros demandeurs de bois chers, tant pour le bâtiment que pour la marine. La mise en valeur des forêts du Pays de Sault doit dès lors se comprendre dans le cadre d'une spécialisation de l'espace régional : le sapin y est favorisé au moment où d'autres secteurs pyrénéens, comme l'Ariège, disposant de communications peu aisées avec l'aval, mais riches en fer, sont dotées d'innombrables forges pour l'approvisionnement desquelles le hêtre est préféré, donc favorisé.

Pour que le Pays de Sault produise du sapin, il est nécessaire de mettre ses forêts à l'abri des interventions jugées désordonnées des populations locales. Comme, par ailleurs, il faut une centaine d'années pour créer une belle sapinière et comme les marchands recherchent le profit immédiat et refusent d'investir sur le long terme, l'État doit intervenir pour protéger la forêt.

Les officiers forestiers s'efforcent d'agir sur l'espace forestier et sur le rapport hêtre/sapin en ayant recours à des mesures juridiques, techniques et "sociales». Ils font reconnaître les droits du roi face aux seigneurs et aux communautés usagères ; ils créent l'espace forestier, qu'ils délimitent par des arpentages et fixent par des abornements, ce qui explique la permanence de nombreuses formes forestières et, semble-t-il, de la superficie totale du $X V I I^{e}$ siècle au début du $X X^{e}$, à la veille de la période de réenrésinement actuelle. Ils écartent le bétail de la forêt en créant un espace pastoral. Ils éliminent le hêtre en arrachant les jeunes plants et, surtout, en favorisant son charbonnage. L'élimination du hêtre réussit parfois si bien que, vers la fin du XVIII ${ }^{e}$ siècle, les habitants de certains villages devaient utiliser le sapin comme bois de chauffage.

D'autres mesures concernent directement le sapin : les officiers réglementent sa coupe, martèlent les pieds avant la coupe et les pièces après, surveillent l'exploitation dans les forêts, les passages de bois à Quillan ; ils réglementent son commerce en fixant les dimensions des bois de marine et de construction, en fixant le 
nombre et l'emplacement des moulins à scie ; ils surveillent ces établissements. Enfin, les officiers cherchent à favoriser les fortes densités et les sapins "bien-venants", ceux qui ne présentent qu'une tige, droite, par pied. Ils comprennent, en outre, que les deux aspects sont liés.

Les mesures «sociales » ont pour but de limiter et de contrôler l'accès dans les forêts, en réglementant les usages, en surveillant les artisans et les marchands qui sont rendus responsables des dégâts survenus dans leurs exploitations, en favorisant les marchands solvables. La pratique des ventes aux enchères joue dans ce sens un rôle fondamental. Elle introduit dans le commerce les principes de l'économie libérale avec toutes les conséquences sociales de l'application de la libre concurrence entre les marchands et les artisans, entre les marchands eux-mêmes.

Au XVIIle siècle, la forêt s'est améliorée. L'époque des grands défrichements est révolue. Les défrichements ne portent plus que sur de petites superficies et si les forestiers dénoncent encore souvent ces pratiques, il semble bien qu'ils confondent, en fait, artigues et défrichements. La végétation forestière a regagné du terrain dans les secteurs de Coumefrède et de Picaussel. De nombreux indices témoignent de la vitalité du sapin. Dans la plupart des forêts, les jeunes plants de moins de trente ans sont en grande quantité. Cet aspect est particulièrement sensible dans les forêts de Coumefrède et de Picaussel, dont l'exploitation peut reprendre dans la seconde moitié du siècle.

L'exploitation elle-même ne semble pas dépasser les possibilités des forêts puisque, dans l'ensemble, les coupes annuellement réalisées sont inférieures aux propositions d'aménagement faites par les forestiers lors des réformations (cf. annexe). La mévente des bois, à la fin du XVII e siècle et au début du XVIIIe, n'a pu que contribuer à l'amélioration. Les densités ont augmenté, on compte de 350 à 400 sapins de plus de $30 \mathrm{~cm}$ de diamètre dans la forêt de Callong, plus de 300 dans les plus belles parties des forêts de Canelle, Fenelle et Panicas, I00 à I50 dans les plus belles parties des autres forêts. Il est vrai que, dans ces mêmes forêts, des triages nombreux sont encore plantés d'un taillis de hêtres parsemé de quelques rares sapins; il est vrai, surtout, que cette amélioration est toute relative puisque l'exploitation reste plus faible que celle qui est pratiquée actuellement. La forêt de Callong a donné, de 1740 à 1790, une moyenne annuelle de $800 \mathrm{~m}^{3}$ de bois ; elle en a fourni 1850 en 1973 et si les moulins à scie ne travaillaient que trois à six mois par an, c'était moins pour des raisons techniques auxquelles on aurait pu remédier que pour des raisons d'approvisionnement.

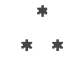

Dès le début de la réformation de 1666, les usagers ont perdu le droit de "jouir de la forêt comme de leur propre chose ". Les usages sont limités « au bois mort et sec en estant et gisant " pour le chauffage et au pâturage du seul bétail local. Enfin, bien que l'usage au bois de réparation soit supprimé, les habitants du Pays de Sault obtiennent la possibilité d'acquérir leurs bois de réparation à un prix inférieur à celui du marché.

Mais, désormais, seuls ceux qui achètent le bois lors des ventes aux enchères ont le droit de fabriquer les menus objets de la vie quotidienne. Ainsi, les artisans, peu nombreux à l'époque de Louis de Froidour, voient leur nombre augmenter et leurs activités se diversifier. Mais leur indépendance disparaît progressivement an cours du XVIII ${ }^{e}$ siècle puisque, par le biais des enchères, les marchands portent les bois qui leur sont destinés à des prix qui leur deviennent inaccessibles. Les artisans tombent ainsi dans la dépendance des marchands ; ils sont prolétarisés.

Les bûcherons subissent un sort identique. Eux qui, avant la réformation de 1666 , coupaient, sans demander la moindre permission, les arbres qu'ils choisissaient, doivent louer leurs services aux marchands adjudicataires, seuls responsables des exploitations.

Les marchands bénéficient ainsi, à la fois, de la réorganisation administrative du marché du bois et de l'amélioration de la sapinière. Certes, l'accroissement du volume global des bois mis en vente dans le cadre de la maîtrise de Quillan reste, malgré tout, modéré, mais les sapins sont plus beaux et disponibles dans des lieux d'accès plus facile ; surtout, la demande, parfois en vive hausse, entraîne un accroissement du prix de vente du sapin. Ce prix est passé de I livre en moyenne vers 1670 à 10 livres et plus à la fin du XVIII ${ }^{c}$ siècle, atteignant même les 30 livres dans la forêt de Callong, la plus belle et la plus proche de Quillan. Les marchands qui doivent débourser quelques centaines de livres vers 1670 pour obtenir l'adjudication des coupes dans une 


\section{FRUHAUF}

forêt, sont obligés d'en débourser quelques milliers un siècle plus tard. Comme, bien souvent, le même marchand se porte adjudicataire de plusieurs coupes, le montant de ses acquisitions peut alors dépasser les 20000 livres.

Il ne nous est pas possible, faute de données quantitatives, d'évaluer le rôle joué dans ce processus d'accumulation par le commerce du bois vers l'aval et par l'exploitation du personnel. Tout au plus, on peut penser que le grand nombre d'opérations nécessaires pour exploiter le bois (coupe, transport, façon) permettent aux marchands de justifier des augmentations de prix, qui sont en fait bien supérieures aux frais réellement engagés. La croissance du capital a surtout été favorisée, avant 1740 , par la possibilité laissée aux marchands de choisir en forêt les arbres qui leur conviennent et par la complaisance, voire la complicité des officiers à leur égard : adjudications de coupes importantes pour de longues durées sans révision des prix, coupes supérieures au nombre des sapins adjugés, modification des contrats d'exploitation après l'adjudication.

Mais tous les marchands ne profitent pas au même titre de ces nouvelles possibilités. Les adjudications les plus importantes restent entre les mains d'une poignée de marchands, les " négociants", tandis que de petits marchands se partagent les ventes d'intérêt secondaire.

Les petits marchands sont installés dans les villages du Pays de Sault et ils exploitent les forêts proches de leurs villages, le plus souvent des forêts d'accès difficile ou fournissant des bois de faible valeur. Les grands marchands sont établis essentiellement à Quillan, accessoirement à Espéraza. II arrive que des marchands extérieurs à la maîtrise de Quillan, installés dans les grandes villes du Bas-Languedoc, participent directement aux adjudications. Il se peut qu'en fait les marchands de Quillan jouent le rôle d'intermédiaires entre la forêt et ces négociants. Mais notre documentation n'est pas assez explicite pour que nous puissions parler de dépendance économique.

Nous ne connaissons effectivement de ces marchands que les sommes qu'ils investissent ou sont prêts à investir dans le commerce du bois. Sur leur personnalité, la nature et le détail de leurs opérations commerciales et financières, nous n'avons que des données fragmentaires et superficielles. Nous percevons surtout l'attitude de ces familles face au foncier : dès le $X V I^{e}{ }^{e}$ siècle, les marchands possèdent des vignobles; au XVIII ${ }^{\mathrm{e}}$ siècle, ils sont propriétaires de la plupart des maisons de Quillan; ils achètent des terres nobles.

Ces marchands constituent ainsi une bourgeoisie locale qui jouit du monopole d'une ressource d'intérêt régional et qui s'appuie, pour mieux assurer sa domination, sur ses liens de parenté, inévitables dans une si petite ville, avec les officiers de la maîtrise.

Cette société, moderne par ses structures, vit dans un calme social relatif. Certes, il y a des révoltes, mais elles sont individuelles et non sociales; il y a des tensions sur les problèmes du pâturage, surtout des défrichements, mais elles n'éclatent pas; il y a enfin une délinquance qu'on peut qualifier de primaire ( $\mathrm{vol}$ de bois, pâturage dans un espace interdit...). Tous ces éléments peuvent être l'expression d'un malaise social; on ne trouve pas pour autant l'expression d'un refus à l'égard de la forêt ou du sapin. Même au XIXe siècle, au moment de la Guerre des Demoiselles en Ariège, le Pays de Sault fut relativement calme.

Le sapin fournit du travail. Il faut une maind'œuvre nombreuse pour le bûcheronnage, le transport, le sciage. Cette main-d'œuvre vit dans les villages, sans qu'il soit possible de faire la part des "spécialistes " et des paysans, travailleurs occasionnels en forêt. Le travail du bois fournit, peut-être, à cette population des ressources qui lui permettent de supporter et d'accepter les entraves apportées à son mode de vie traditionnel.

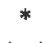

Cet aménagement, engagé dès les $X V I^{e}{ }^{e}$ et XVlI]e siècles, s'est poursuivi depuis lors, conduit par une même volonté, soutenu par des $m$ ens sans cesse accrus, surtout par une connaissance toujours améliorée des possibilités offertes par la nature. Mais si notre étude montre l'insuffisance, pour la compréhension des milieux, d'une analyse strictement naturaliste, elle montre aussi l'insuffisance des analyses qui n'introduisent que l'Homme dans les milieux naturels. Cette démarche plus actuelle masque en fait les conflits sociaux que peut susciter toute politique d'aménagement de l'espace. La nature offre des potentialités - hêtraie-sapinière ou dominance d'une des deux formations dans le cadre 
territorial de notre étude - le groupe qui a la maîtrise des techniques, le groupe socialement dominant choisit parmi ces potentialités la plus conforme à ses intérêts.

\section{FRUHAUF \\ Institut de Géographie UNIVERSITÉ DE TOULOUSE-LE-MIRAIL \\ 109 bis, rue Vauquelin 31081 TOULOUSE CEDDEX}

\section{ANNEXE}

DONNÉES COMPARATIVES SUR L'EXPLOITATION DU SAPIN DANS QUELQUES FORETS DU PAYS DE SAULT aux $X V I I^{e}-X V I I I^{e}$ siècles et aujourd'hui
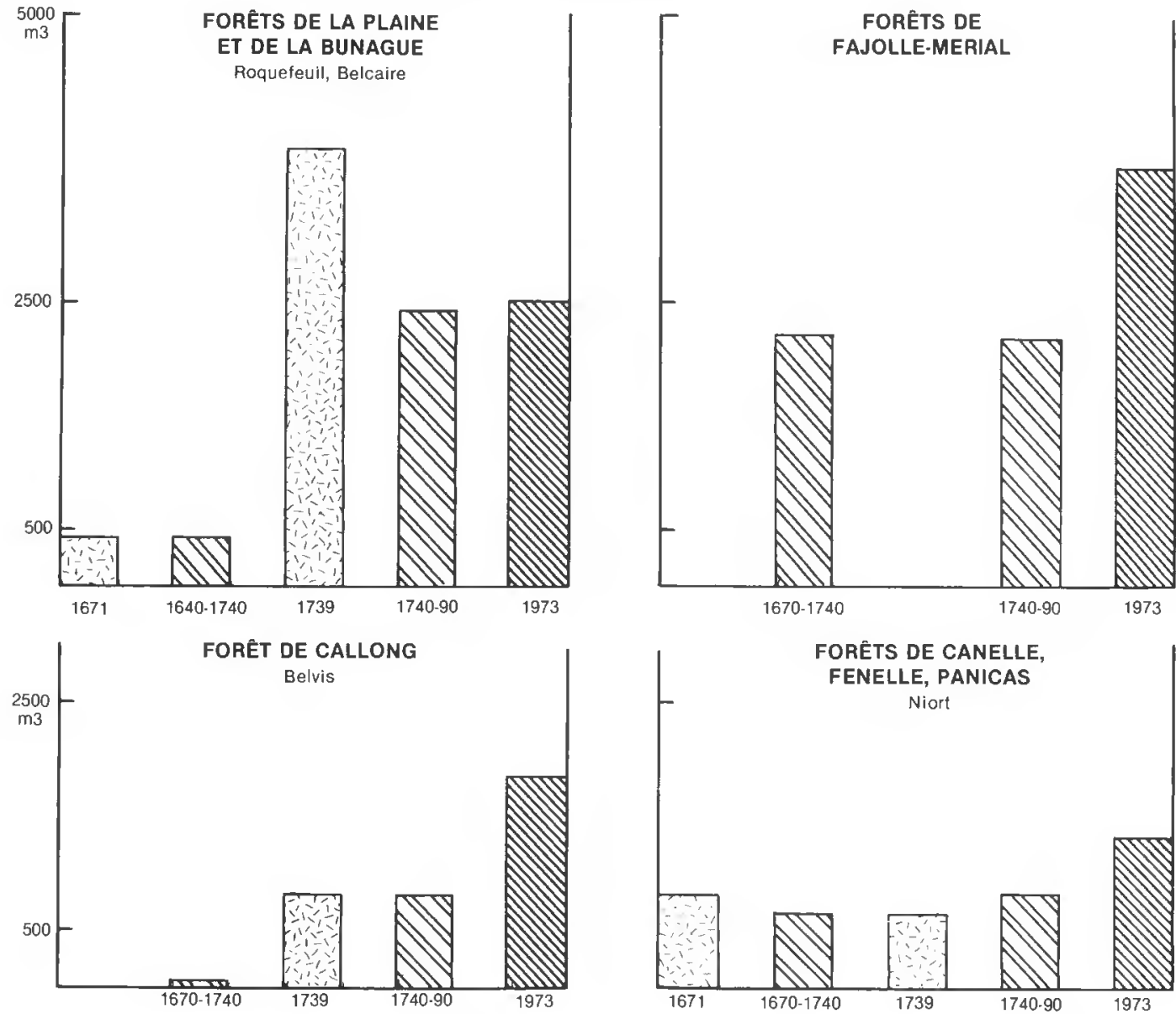

Propositions pour l'exploitation des foréts en 1671 et 1739 (1)

Coupes mises en vente, le 16 octobre 1973 à Quillan pour étre exploitées (2) en 1974

(1) Nous avons transformé en $m 3$ les pieds de sapins sur la base : 1 sapin $=4 \mathrm{~m}^{3}$

(2) Les chiffres sont fournis en $\mathrm{m} 3$. 